\title{
HIGH-T $_{c}$ SUPERCONDUCTIVITY: PRESENT STATUS OF FUNDAMENTAL RESEARCH AND APPLICATIONS
}

\author{
H. RIETSCHEL \\ Kernforschungszentrum Karlsruhe, Institut für Nukleare Festkörperphysik, Postfach 3640, D-7500 \\ Karlsruhe, FRG
}

(Received November 15, 1992; in final form December 16, 1992)

\section{INTRODUCTION}

In 1986, Bednorz and Müller discovered superconductivity around $\mathrm{T} \sim 30 \mathrm{~K}$ in a ceramic compound of composition $(\mathrm{La}, \mathrm{Ba})_{2} \mathrm{CuO}_{4}[1]$. Soon afterwards, superconductivity below $T_{c}=92 \mathrm{~K}$ was found in a related compound of composition $\mathrm{YBa}_{2} \mathrm{Cu}_{3} \mathrm{O}_{7}$. For the first time, the possibility was opened up to maintain superconductivity at $\mathrm{T}=77 \mathrm{~K}$, the boiling point of liquid nitrogen $\left(\mathrm{LN}_{2}\right)$.

The discovery of these "high-temperature superconductors" (HTSCs) initiated an unprecedented boom in research aiming at both the physical understanding of HTSC and the technical application of these new materials. In particular the latter point, namely the prospect of developing a new superconductivity technology based upon $\mathrm{LN}_{2}$ cryogenics, has attracted wide interest and initiated a wealth of new R\&D activities all over the world. In what follows, we will only briefly comment on the physical understanding of the phenomenon of HTSC but mainly concentrate on a review of the present status of applications for these new and challenging materials.

\section{PHYSICAL UNDERSTANDING}

As of the physical origin of HTSC, a good guide is provided by the classical superconductors such as, e.g., $\mathrm{Hg}, \mathrm{Nb}$ or $\mathrm{Al}$ which because of their low transition temperatures must all be cooled with liquid $\mathrm{He}^{4}(4.2 \mathrm{~K})$. In these materials, the phenomenon of superconductivity is well understood and basically formulated in the theory of Bardeen, Cooper, and Schrieffer (BCS-theory) [2].

The central point of this theory is pairing of electrons. These Cooper pairs consist of two electrons with opposite spin and momentum which then, as a whole, condense into a so-called macroscopic quantum state. Pairing requires an attractive interaction between the partners, and in classical superconductors, this attraction 
between the electrons is mediated by the polarizability of the positively charged ionic lattice of the metal (Fröhlich interaction [3]).

Regarding now the HTSCs, there is unambiguous experimental evidence that the electrons also condense into a macroscopic quantum state as pairs. A particularly convincing proof for this is the observation of quantization of magnetic flux in tiny cylinders of HTSC material [4], where the flux quantum is $h / 2 \mathrm{e}(\mathrm{h}$ : Planck's constant, e: elementary charge) as required for pairs. However, in contrast to the classical superconductors, the nature of the attractive interaction behind this pairing is still unclear in the HTSCs. Besides the Fröhlich interaction mentioned above, many other mechanisms, in particular magnetic interactions, are presently discussed as possible alternative explanations [5].

\section{HTSC FAMILIES: CHARACTERISTICS AND PREPARATION}

Let us now turn to the present status of applications for the HTSCs. Virtually all applications of high- $\mathrm{T}_{\mathrm{c}}$ cuprate superconductors are restricted to cuprates with superconducting transition temperatures $T_{c}>77 \mathrm{~K}$, thus allowing the maintenance of superconductivity by cooling with liquid nitrogen. These materials may roughly be grouped into four families. The most prominent members of these families are listed in Table 1 , together with their short-hand notations and the maximum $T_{c}$ reached up to the present time [6].

With respect to applications, the most important (and detrimental) characteristics of these compounds are the high anisotropy of the critical current density, $\mathrm{J}_{\mathrm{c}}$, and the very short coherence length, $\xi, 2 \mathrm{~A}<\xi<20 \mathrm{~A}$. In polycrystalline samples, this leads to weak-link behavior at the grain boundaries, with $\mathrm{J}_{\mathrm{c}}$ strongly reduced in comparison to its intragrain value ("granularity"). This is the actual reason that in ceramic samples, $J_{c}(77 \mathrm{~K}, 0 \mathrm{~T})$ is always found to be below $\sim 1000 \mathrm{~A} / \mathrm{cm}^{2}$, and in magnetic fields, is reduced even further. Thus for most applications, ceramic (or "granular") HTSCs are completely useless.

TABLE I

\begin{tabular}{|c|c|c|c|}
\hline \multirow{2}{*}{$\begin{array}{l}\text { Formula } \\
\mathrm{REBa}_{2} \mathrm{Cu}_{3} \mathrm{O}_{7} \\
(\mathrm{RE}=\text { "Rare Earth" = Y,Eu,Gd, . .) } \\
\mathrm{Bi}_{2} \mathrm{Sr}_{2} \mathrm{Ca}_{n-1} \mathrm{Cu}_{\mathrm{n}} \mathrm{O}_{2 \mathrm{n}+4} \\
(+\mathrm{Pb} \text { doping})^{+}\end{array}$} & \multirow{2}{*}{$\begin{array}{l}\text { Short hand } \\
\text { "RE BCO" or " } 123 \text { " } \\
\text { "BSCCO" or "Bi- } 22(n-1) n "\end{array}$} & \multicolumn{2}{|c|}{ Maximum $T_{c}$} \\
\hline & & $\begin{array}{r}90 \mathrm{~K} \\
122 \mathrm{~K} \\
90 \mathrm{~K}\end{array}$ & $\begin{array}{l}(\mathrm{YBCO}) \\
(\mathrm{Bi}-2212) \\
(\mathrm{Bi}-2223) \\
(\mathrm{Bi}-2234)\end{array}$ \\
\hline $\mathrm{Tl}_{2} \mathrm{Ba}_{2} \mathrm{Ca}_{n-1} \mathrm{Cu}_{n} \mathrm{O}_{2 n+4}$ & "TBCCO" or "Tl-22(n-1)n" & $\begin{array}{l}110 \mathrm{~K} \\
127 \mathrm{~K} \\
119 \mathrm{~K}\end{array}$ & $\begin{array}{r}(\mathrm{Tl}-2212) \\
(\mathrm{Tl}-2223) \\
(\mathrm{Tl}-2234)\end{array}$ \\
\hline $\begin{array}{l}\mathrm{Tl}_{1} \mathrm{~A}_{2} \mathrm{Ca}_{\mathrm{n}-1} \mathrm{Cu}_{\mathrm{n}} \mathrm{O}_{2 \mathrm{n}+3} \\
(\mathrm{~A}=\mathrm{Sr}, \mathrm{Ba})\end{array}$ & "Tl-12(n-1)n" & $\begin{array}{r}90 \mathrm{~K} \\
122 \mathrm{~K} \\
122 \mathrm{~K} \\
110 \mathrm{~K}\end{array}$ & $\begin{array}{l}\text { (Tl-1212) } \\
\text { (Tl-1223) } \\
\text { (Tl-1234) } \\
\text { (Tl-1245) }\end{array}$ \\
\hline
\end{tabular}


At present, two strategies are pursued to reach higher $J_{c}$ values in cuprate superconductors:

i) preparation of epitaxial films and

ii) preparation of highly textured bulk samples.

Both methods aim at a reduction in the number of grain boundaries by singlecrystallinity and at the alignment of the $\mathrm{CuO}_{2}$-planes in the most favorable direction, i.e., parallel to the transport currents.

Epitaxial films are grown on single-crystalline substrates like $\mathrm{SrTiO}_{3}, \mathrm{LaAlO}_{3}$ or $\mathrm{Al}_{2} \mathrm{O}_{3}$ with matching cuts. Currently, the growth and processing procedures for YBCO-films are the furthest advanced. Various techniques such as laser ablation, sputtering or MOCVD allow the reproducible preparation of films with $T_{c}>90 \mathrm{~K}$ and $\mathrm{J}_{\mathrm{c}}(77 \mathrm{~K}, 0 \mathrm{~T})>10^{6} \mathrm{~A} / \mathrm{cm}^{2}$ [7]. Meanwhile, high- $\mathrm{J}_{\mathrm{c}}$ films based on $\mathrm{Tl}$ - and $\mathrm{Bi}-$ HTSCs have been prepared, but their $\mathrm{J}_{\mathrm{c}}$-values are much more sensitive to applied magnetic fields.

Texturing of HTSC bulk-samples, wires or tapes can be achieved by a number of methods: for example, crystallization from the melt in a temperature gradient or mechanical treatments like pressing and rolling. At the moment, the main progress in melt texturing has been achieved for YBCO bulk samples, while for future applications in conductors, tapes or wires containing textured $\mathrm{Bi}-2212$ or Bi-2223 material seem to be particularly promising.

\section{APPLICATIONS OF FILMS}

Passive microwave devices (filters, delay lines) designed in stripline technology based on YBCO and Tl-2223 thin-films $(\mathrm{d}<3000 \mathrm{~A})$ will form the first HTSC applications [8]. As a result of their electronic energy gap, HTSCs exhibit much lower rf-losses than normal conductors, at least in the frequency range below about $100 \mathrm{GHz}$. Therefore, superconducting resonators-the basic component of these devices-can be made considerably smaller and lighter than their normal conducting counter-parts for a given performance. Superconducting multipole filters in the $10 \mathrm{GHz}$ range have already been put up for sale.

Similar arguments hold for small microwave antennas, which are also composed of resonators. Miniaturized planar antennas manufactured from HTSC thin films show losses considerably reduced in comparison to normal conducting models of equal size [9]. This is particularly advantageous if highly directional antenna arrays are considered. Only recently, a group antenna consisting of 64 Tl-HTSC thin-film elements was successfully tested in the USA.

Not only the lower rf losses but also the lower dispersion of short pulses in superconducting striplines is an essential feature for certain applications, e.g., as the interconnects between semiconducting chips in ultra high-speed computers. In those cases where the computers are already cooled by liquid nitrogen, such a semiconductor-superconductor hybrid technology does not require additional cryogenics and is thus particularly straightforward. A USA research program has been initiated that aims at the development of such HTSC multi-chip modules. 
HTSC thin films can also form the basis of highly sensitive IR sensors. Making use of the steep $R(T)$-characteristic at the superconducting transition temperature, $\mathrm{T}_{\mathrm{c}}$, HTSC bolometers are the most sensitive IR detectors in the far infrared, where semiconductors with sufficiently small energy gap are not available. Work on integration of many of such pixels into position sensitive IR detectors has already been initiated [10].

Finally, let us briefly discuss HTSC thick film applications $(\mathrm{d}>1 \mu \mathrm{m})$. Lifting the requirement of epitaxy, much simpler preparation methods (e.g., electrophoresis, sol-gel, spreading of pastes) may be applied which lend themselves also to large or curved surfaces. Although with respect to critical currents and rf losses, these polycrystalline thick films are by far inferior to epitaxial thin films, they may still be used advantageously for coating rf devices, a good example being the HTSCcoated cavity for a hydrogen maser [11].

However, the main application of HTSC thick films is magnetic shielding. Several Japanese companies have already developed shielding chambers based on the use of Bi-HTSC thick films. A system consisting of a cylindrical chamber $40 \mathrm{~cm}$ long and $15 \mathrm{~cm}$ in diameter, which attenuates magnetic background fields by a factor of $10^{4}$ to $10^{5}$ is already on the market.

\section{TEXTURED CURRENT LEADS AND BULK SAMPLES}

Regarding the development of HTSC current leads, present interest is focused on $\mathrm{Bi}-\mathrm{HTSC}$ wires or tapes. Here, the $\mathrm{Bi}-2212$ phase offers extraordinarily favorable conditions for its use in helium-cooled high-field magnet coils: with $\mathrm{J}_{\mathfrak{c}}(4.2 \mathrm{~K}, 25$ $\mathrm{T})=140,000 \mathrm{~A} / \mathrm{cm}^{2}$, its critical current density in high magnetic fields surpasses that of any classical superconductor (e.g., $\mathrm{NbTi}$ or $\mathrm{Nb}_{3} \mathrm{Sn}$ ). For cooling under liquid nitrogen, on the other hand, the $\mathrm{Bi}-2223$ phase is superior: with $\mathrm{J}_{\mathrm{c}}(77 \mathrm{~K}, 0 \mathrm{~T}) \sim$ $50,000 \mathrm{~A} / \mathrm{cm}^{2}$, it is suitable for use in high-current leads at low fields; in high fields, however, its $\mathrm{J}_{\mathrm{c}}$ rapidly decreases [12].

To manufacture such wires or tapes, silver tubes filled with HTSC powder or silver tapes spread with HTSC pastes are subjected to consecutive steps of tempering, followed by pressing or rolling. The role of the silver is not yet clear: it is assumed that it not only supports the alignment of HTSC crystallites but also helps to avoid cracking as a result of its plasticity. By adding finely dispersed $\mathrm{Ag}$ powder to the precursor material, wires have been produced that can be stretched up to $2 \%$ without irreversible degradation of $J_{c}$. In view of all this progress, industrial production and technical use of HTSC current leads may be expected in the near future.

Until now, all fabricated magnet coils based on HTSC materials have only been "demonstrators" and are without practical use. There is one major hurdle that must yet be overcome, and this is the phenomenon of flux creep. Even in low magnetic fields (e.g., self field of the transport current), thermally activated flux lines move under the Lorentz force of the transport current, thus leading to dissipation. Even in the Bi-HTSC tapes and wires described above, flux creep still remains a problem. This excludes operation of the magnet without external current 
source (persistent mode). Very recently it was discovered that in the Tl-1223 phase, magnetic flux can be pinned much more effectively than in the Bi-based HTSC so that flux creep sets in only above $\mathrm{B} \sim 10 \mathrm{~T}$ [13]. In fact, short Tl-1223 wires with $\mathrm{J}_{\mathrm{c}}(77 \mathrm{~K}, 1,5 \mathrm{~T})=8000 \mathrm{~A} / \mathrm{cm}^{2}$ have already been produced by similar techniques as were developed for the Bi-HTSCs.

By means of a rather slow procedure that is commonly called "melt texturing" and which consists of a sequence of melting and cooling steps, highly textured plates or disks of YBCO can be produced with up to several hundred grams of weight. In these melt-textured YBCO samples, magnetic flux is pinned very efficiently, and high critical currents can be reached at high fields. Presently, typical values are $J_{c}(77 \mathrm{~K}, 1 \mathrm{~T})=30,000 \mathrm{~A} / \mathrm{cm}^{2}$ and $J_{c}(77 \mathrm{~K}, 5 \mathrm{~T})=15,000 \mathrm{~A} / \mathrm{cm}^{2}$. As a result of its time consuming nature, this process is hardly suited for making wires or tapes, but the melt-textured disks or plates do offer a very interesting potential application as magnetic bearings. If such a sample is cooled below $T_{c}$ in the field of a permanent magnet, magnetic flux gets frozen in. Due to the strong flux pinning, any attempt to displace the magnet will result in a restoring force (present maximum: $\sim 10 \mathrm{~N} / \mathrm{cm}^{2}$ ) that allows fabrication of stable passive magnetic bearings [14]. Heavy-duty bearings for high loads (up to $100 \mathrm{~kg}$ ) as well as high-speed bearings reaching 500,000 rpm have already been designed and realized based on melttextured YBCO. One particularly interesting application of superconducting magnetic bearings could be their use with energy storing flywheels.

A further application of HTSCs in energy technology is their use in current limiters. In this case, a superconducting line is pushed by shock currents or shock fields into its highly resistive normal state, thus limiting possible short circuit currents in the network. However, for more demanding applications in energy technology like superconducting transformers, generators or magnetic energy storage devices, HTSC materials are presently far from being worth discussing.

\section{JOSEPHSON CONTACTS}

A number of potential applications for superconductivity results from the Josephson effects that occur at two weakly coupled superconductors (the Josephson contact). The high sensitivity of the dc Josephson current to magnetic fields is exploited in SQUIDs, which are presently by far the most sensitive detectors of magnetic fields. Designed in classical Nb-technology, SQUIDs are widely used in science and more recently even in medical diagnostics for both magnetoencephalography and cardiography. Besides SQUIDs, oscillators and mixers for ultrahigh frequencies are further devices where $\mathrm{Nb}$ Josephson contacts are employed advantageously. For the ac Josephson effect, the realization of high-precision voltage standards is the most important application.

Various methods have been developed to prepare Josephson contacts based on HTSC thin films. These are reviewed elsewhere together with special designs for HTSC SQUIDs and their properties such as sensitivity and noise figures. Summarizing these discussions one can state that now, HTSC SQUIDs already come close to commercially fabricated Nb SQUIDs in their performance. The progress 
achieved in this field is perhaps best illustrated by the on-chip integration of SQUID and flux transformer by CONDUCTUS [15]. Although not yet optimized in sensitivity and noise figure, with its 15 different HTSC and buffer layers, this device represents the state-of-the-art of HTSC thin-film technology. Similarly integrated SQUID chips in somewhat simpler design are already offered at low price for practical training of students.

SQUIDs may also be considered an intermediate step towards digital electronics based on Josephson contacts. There appears to be decreasing support for the concept of using hysteretic Josephson contacts as flip-flops in fast computers. This concept, which has been pushed ahead in the past, particularly by the Japanese, does not offer much faster clock speeds than advanced semiconductor technology. Much faster superconducting logical components may be expected from the various concepts based on rapidly moving single flux quanta ("RSFQ"), but these new and futuristic ideas have yet to be realized in classical $\mathrm{Nb}$ technology, let alone in HTSC! What can be expected in the near future, however, is the development of small scale integrated Josephson circuitry (e.g., fast A/D converters) to be used, for instance, as the read-out electronics of a SQUID located on the same cold chip $[16]$.

\section{CONCLUSIONS}

Within a remarkably short time, HTSCs have reached a stage of development for which classical superconductors have needed a much longer period. Although some of the first dreams and hopes have turned out to be unrealizable at least in the near future (in particular energy technology), the already realized ideas such as SQUIDs, microwave components, or magnetic bearings demonstrate that HTSCs will find wide applications in future technologies.

\section{REFERENCES}

1. J.G. Bednorz and K.A. Müller, Z. Physik B 64 (1986) 189

2. J. Bardeen, L.N. Cooper, and J.R. Schrieffer, Phys. Rev. 108 (1957) 1175

3. H. Fröhlich, Phys. Rev. 79 (1950) 845

4. C.E. Gough et al., Nature 326 (1987) 855

5. H. Rietschel, Phys. Bl. 46 (1990) 419

6. K. Wasa et al., J. Mat. Res. 6 (1991) 1595

7. J. Geerk et al., Mat. Sci. Rep. 4 (1991) 193

8. R.W. Ralston, Supercond. Sci. Technol. 4 (1991) 386

9. H. Chaloupka et al., IEEE Trans. Microwave Theory Tech. 39 (1991) 1513

10. S. Verghese et al., IEEE Trans. Magn. 27 (1991) 3077

11. D. Opie et al., IEEE Trans. Magn. 27 (1991) 2944

12. K. Sato et al., J. Appl. Phys. 70 (1991) 6484

13. R.S. Liu et al., Appl. Phys. Lett. 60(8) 24.2.1992

14. M. Murakami et al., IEEE Trans. Magn. 27 (1991) 1479

15. R. Simon, Physics Today, June 1991, 64

16. K.K. Likharev et al., IEEE Trans. Appl. Supercond. 1 (1991) 3 

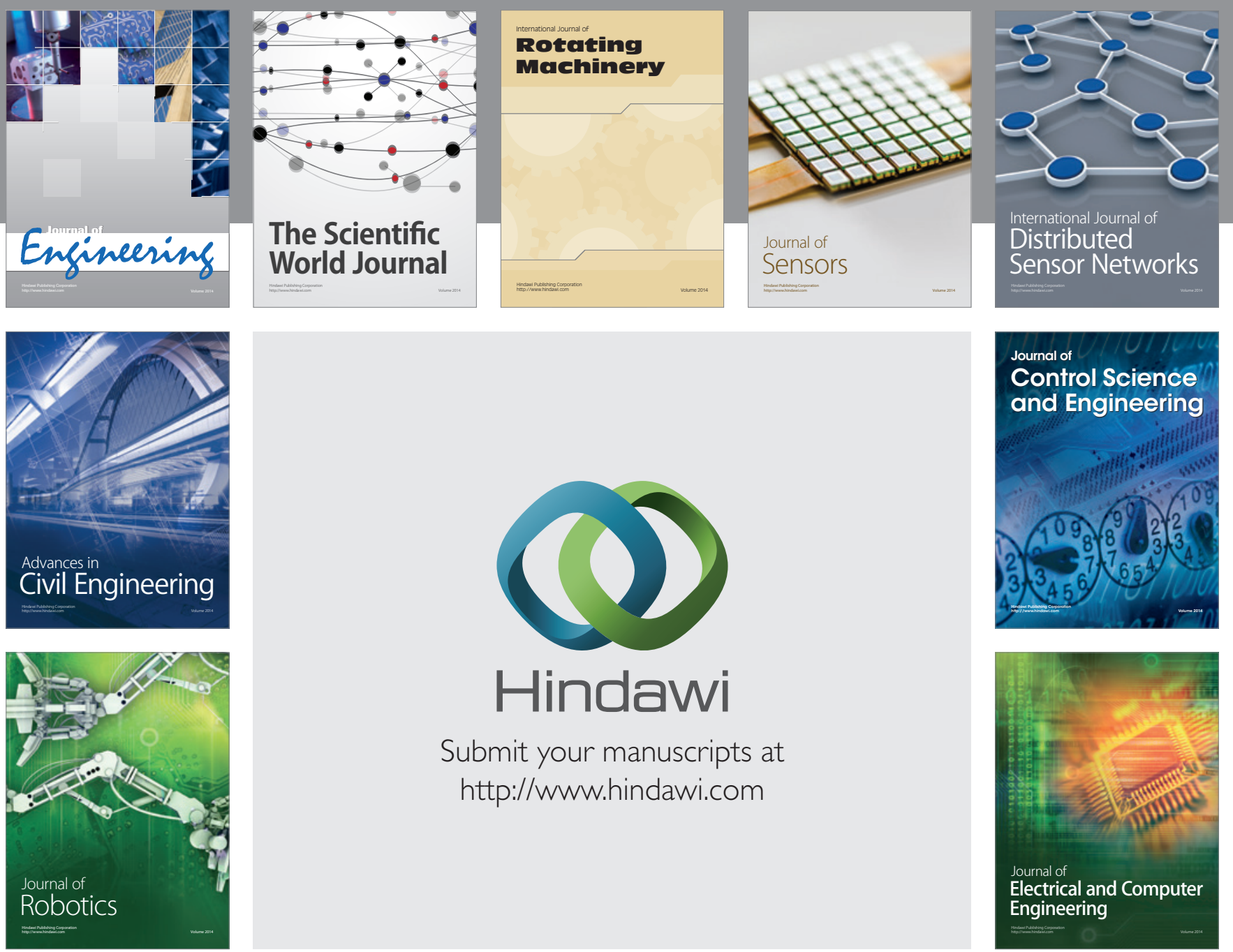

Submit your manuscripts at

http://www.hindawi.com
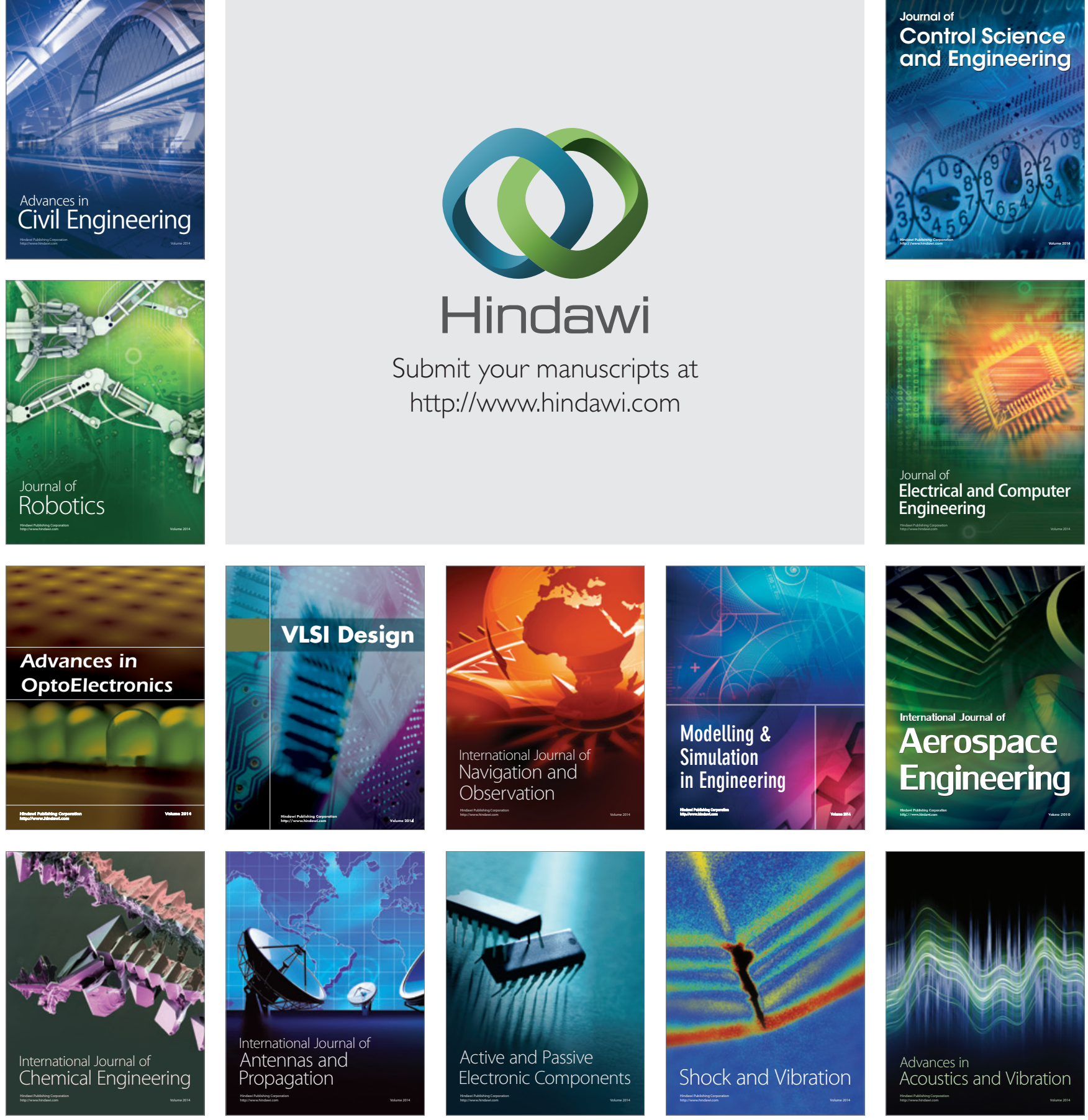